\title{
BMJ Open Exploring similarities and differences in hospital adverse event rates between Norway and Sweden using Global Trigger Tool
}

\author{
Ellen Tveter Deilkås, ${ }^{1,2}$ Madeleine Borgstedt Risberg, ${ }^{3}$ Marion Haugen, ${ }^{4}$ \\ Jonas Christoffer Lindstrøm, ${ }^{2}$ Urban Nylén, ${ }^{5}$ Hans Rutberg, ${ }^{6,7}$ Soop Michael ${ }^{5}$
}

To cite: Deilkås ET, Risberg MB, Haugen M, et al. Exploring similarities and differences in hospital adverse event rates between Norway and Sweden using Global Trigger Tool. BMJ Open 2017;7:e012492. doi:10.1136/bmjopen-2016012492

- Prepublication history for this paper is available online. To view these files please visit the journal online (http://dx.doi.org/10.1136/ bmjopen-2016-012492).

Received 3 May 2016 Revised 18 January 2017 Accepted 23 January 2017

CrossMark

For numbered affiliations see end of article.

Correspondence to Dr Ellen Tveter Deilkås; elde@ahus.no

\section{ABSTRACT}

Objectives: In this paper, we explore similarities and differences in hospital adverse event (AE) rates between Norway and Sweden by reviewing medical records with the Global Trigger Tool (GTT).

Design: All acute care hospitals in both countries performed medical record reviews, except one in Norway. Records were randomly selected from all eligible admissions in 2013. Eligible admissions were patients 18 years of age or older, undergoing care with an in-hospital stay of at least 24 hours, excluding psychiatric and care and rehabilitation. Reviews were done according to GTT methodology.

Setting: Similar contexts for healthcare and similar socioeconomic and demographic characteristics have inspired the Nordic countries to exchange experiences from measuring and monitoring quality and patient safety in healthcare. The co-operation has promoted the use of GTT to monitor national and local rates of AEs in hospital care.

Participants: 10986 medical records were reviewed in Norway and 19141 medical records in Sweden.

Results: No significant difference between overall AE rates was found between the two countries. The rate was $13.0 \%(95 \% \mathrm{Cl} 11.7 \%$ to $14.3 \%)$ in Norway and $14.4 \%(95 \% \mathrm{Cl} 12.6 \%$ to $16.3 \%)$ in Sweden. There were significantly higher AE rates of surgical complications in Norwegian hospitals compared with Swedish hospitals. Swedish hospitals had significantly higher rates of pressure ulcers, falls and 'other' AEs.

Among more severe AEs, Norwegian hospitals had significantly higher rates of surgical complications than Swedish hospitals. Swedish hospitals had significantly higher rates of postpartum AEs.

Conclusions: The level of patient safety in acute care hospitals, as assessed by GTT, was essentially the same in both countries. The differences between the countries in the rates of several types of AEs provide new incentives for Norwegian and Swedish governing bodies to address patient safety issues.

\section{INTRODUCTION}

Interpreting the extent to which patient safety indicators accurately reflect international

\section{Strengths and limitations of this study}

- The samples are drawn from all eligible hospital admissions in both countries in 2013.

- The samples represent $1.9 \%$ of all eligible hospital admissions in Norway and $1.4 \%$ in Sweden.

- This is the first explorative cross-country comparison of adverse event rates and types based on Global Trigger Tool.

- The study does not include demographic data or other patient characteristics.

- The reviewing process differed slightly between the countries and

- inter-rater reliability between review teams across countries was not assessed.

differences in patient safety must be done with caution. Differences may reflect the way that countries report, code and calculate rates of adverse events (AEs) as seen by the Organisation for Economic Co-operation and Development (OECD) indicators. ${ }^{1-3}$ In some cases, higher AE rates may signal more developed patient safety monitoring systems rather than substandard care. In 2007, the Nordic Council of Ministers initiated a project to develop and strengthen Nordic efforts to measure and monitor quality and safety in healthcare. Results of the Nordic project were reported in 2010. ${ }^{4}$ As part of the project, Nordic expert groups were constituted in various fields of patient safety like patient safety culture surveys and Global Trigger Tool (GTT). ${ }^{5}{ }^{6}$ Nordic experiences from using GTT have been reported previously. ${ }^{7}$ The Nordic co-operation and exchange of experiences promoted the use of GTT in Norway and Sweden which, to the best of our knowledge, are among the few countries that have required use of GTT in all hospitals as part of a national government policy. 
A research scan from the Health Foundation has considered GTT to compare well to other approaches, to be relatively sensitive and to identify significantly more AEs compared with self-reporting or other chart audit methods. ${ }^{8}$ GTT produces substantial inter-rater reliability within and between independent internal teams. ${ }^{9} 10$ An evidence scan from the New Zealand Health Quality and Safety Commission considers GTT to be the most accurate and efficient method to identify AEs, although further work is needed to confirm its reliability. ${ }^{11}{ }^{12}$ Most of the studies describing its use are based on large samples from multiple hospitals. ${ }^{12}{ }^{13}$ Although GTT is considered relevant for measuring AEs at the national level, ${ }^{8}$ we are only aware of one publication describing the process of doing so. ${ }^{14}$ This publication presents Norwegian results from 2010 to 2013, while in this study we compare results from 2013 between Norway and Sweden.

Norway and Sweden have similar structural conditions and contexts for healthcare as tax-based funding, similar socioeconomic status, demographic characteristics, publically funded education of healthcare employees and democratic policies pursuing equal access. ${ }^{15}$ Both countries are politically stable and have over decades pursued co-operation and mutual learning. ${ }^{4}$ Sweden is a step ahead regarding quality registers and did many hospital mergers in the mid-1990s, while these happened 10 years later in Norway. ${ }^{16}$ In 2010 when the Norwegian and Swedish national patient safety campaigns were prepared, Sweden had already made a national AE study based on the Harvard Medical Practice Study (HMPS) protocol. ${ }^{17}$ The national patient safety campaign initiatives in the two countries were not co-ordinated, but target areas like, for example, hospital-acquired infections, pressure ulcers and falls were similar. In Norway, GTT was considered a cost-effective method to monitor levels of AEs at hospital level, in relation to the patient safety campaign efforts. Sufficient knowledge and experience was at hand for the purpose. ${ }^{4}{ }^{18}$ Evidence of $\mathrm{AE}$ rates from other countries created an additional momentum in Norway to facilitate, so that the local GTT data could be used to estimate national AE rates. ${ }^{14}$ In the Swedish campaign, it was decided to use GTT rather than the HMPS method as GTT is not just a tool for measurement but also a tool for learning and patient safety improvement work locally. During planning and implementation of the GTT tool, experiences were exchanged in expert meetings and correspondence within the network derived from the Nordic Council of Ministers. In this paper, we explore similarities and differences in hospital AE rates between Norway and Sweden based on GTT in 2013.

\section{Norwegian patient safety campaign}

In 2011, the Norwegian government launched a national patient safety campaign. ${ }^{18}$ The aims were to reduce patient harm, increase knowledge and competence in patient safety and improve patient safety culture. GTT was chosen as the way to do continuous medical record reviews in all acute care hospitals. The patient safety campaign also set requirements for the hospitals to aim at reducing preventable AEs by $20 \%$ during the campaign period, which ended in 2013. To monitor AEs, annual rates were estimated at national level and data at hospital level were plotted in run charts.

\section{Swedish patient safety campaign}

A national initiative to increase patient safety was launched by the government and The Swedish Association of Local Authorities and Regions (SALAR) for the period 2011-2014. ${ }^{19}$ The initiative involved financial incentives and the agreement focused on patient safety culture, hospital-acquired infections, pressure ulcers, prescription of antibiotics and medication errors. As a part of this initiative, all Swedish acute care hospitals have performed medical record reviews according to the GTT method since 2012. Education of review teams and creation of a national database for registration of data from the reviews were included in this initiative.

\section{METHODS}

\section{Medical record review by use of the GTT}

GTT is an internationally recognised and standardised procedure for medical record review to identify and estimate rates and severity of AEs among adult patients in non-psychiatric hospital admissions. ${ }^{6}$ It involves an educated team of two primary reviewers, often nurses, and a physician. A list of criteria (triggers) that indicate a higher probability of AEs is used to identify details in the record that might indicate possible AEs. Readmission to hospital within 30 days after discharge and infection during the hospital stay are examples of triggers. The primary reviewers examine records on their own before they compare results with each other and ultimately validate them with the physician. The identified AEs are then classified regarding type and rated on a 5 -point severity scale (table 1 ).

\section{Sampling}

The data presented in this study were collected separately by independent government-initiated processes in each country. The study was designed after data had been collected. Data from reviews of medical records from hospital admissions in 2013 were used. The reviews

Table 1 Categories used to assess the severity of adverse events (AEs)

E AE contributed to temporary harm to the patient which required intervention

F AE contributed to temporary harm to the patient which required initial or prolonged hospitalisation

G AE contributed to permanent patient harm

$\mathrm{H}$ Intervention was required to sustain life

I AE contributed to patient death 
were based on the GTT methodology on randomly selected medical records. Selection criteria were completed records from admissions of at least 18-year-old patients with in-hospital stays that lasted at least 24 hours. Admissions in psychiatric or rehabilitation departments were excluded. The reviews included the whole period of hospitalisation even if the patients had been treated in different departments during the hospital stay. The admissions that were completed 1 month earlier were eligible for the random selection as specified by the GTT method. The whole population of eligible admissions in each hospital had to be covered by one or more randomly selected samples that could not overlap. For identification of records to review, lists of all eligible admissions were retrieved locally by the electronic administrative system in each hospital, and a random selection of the required number of records was done.

\section{Settings}

In Norway, 21 acute care hospitals (all except one) and two small non-acute care hospitals, with a total of 45 GTT teams, participated in the review. The teams selected at least 10 medical records bimonthly, which two trained registered nurses (RNs) first reviewed separately. They then compared their results and validated them with a physician.

All 63 Swedish acute care hospitals participated. The minimum monthly number of randomly selected admissions reviewed was 40 for university hospitals, 30 for the central county council hospitals and 20 for the small hospitals. Each hospital had its own review team. In some of the university hospitals, more than one team performed the reviews but the results were then pooled into one list for the hospital. An RN searched the records for triggers. After that a team, with one or more $\mathrm{RN}$ and one or more physicians, assessed records with positive triggers, to identify possible AEs and classify them.

Reviewers in both countries were employed in the hospitals where they worked clinically. They were all clinically experienced reviewers although some worked in the quality department, where they sometimes did not do clinical work at the time of the review. Our strategy leans on a study where internal GTT teams found more AEs than external teams. ${ }^{9}$

\section{Translation and validation}

In Norway, a professional translator translated the original GTT white paper to Norwegian in $2010 .^{20}$ It was then slightly modified regarding a few triggers. ${ }^{14} \mathrm{~A}$ protocol with rules for the national co-ordination of the GTT review was provided. The list of frequently asked questions (FAQ) was expanded with experience during the first 6 months of the review, which started in January 2011. The translated original GTT white paper, together with the FAQ list and the protocol, constituted the Norwegian manual.
In 2008, a Swedish translation and adaptation of the GTT method was introduced in some hospitals. The Swedish manual was revised in $2012 .{ }^{21}$ Accordingly, triggers that were seldom found or rarely indicated an $\mathrm{AE}$ were omitted and four new triggers were introduced to increase sensitivity for AEs in non-surgical care. The descriptions of triggers were reformulated and markedly expanded in order to facilitate assessment of the severity of the AEs. ${ }^{22}$

\section{Training and standardisation}

In Norway, training was organised and delivered by the secretariat of the national patient safety campaign, starting in January 2011. The teams were since then trained by a physician specialised in internal medicine, who also provided support by phone and email. The standard 1-day course, according to the GTT manual, included review of five training records. Forty additional medical records were required to be reviewed before participating in the ordinary reviews. Teams were gathered annually, by the secretariat of the national patient safety campaign, at national meetings to present results, cases, and to review and calibrate routines and definitions.

In Sweden, training in the GTT methodology for review teams was initiated in the beginning of 2012 by educational teams appointed by SALAR. Each educational session was led by a physician and a nurse, both experts in GTT, and comprised a combination of lectures and training for 1 day. Support from the central team of educators was continuously available by phone. Meetings for review teams were arranged regionally for calibration and support.

\section{Definitions, categorising and reporting of data}

In both countries, an $\mathrm{AE}$ was defined according to the GTT definition of harm as an 'unintended physical injury resulting from, or contributed to, by medical care that requires additional monitoring, treatment or hospitalisation or that results in death'. ${ }^{6}$ In both countries, AEs were categorised according to severity and type. Severity was categorised according to 'National Coordinating Council for Medication Error Reporting and Prevention (NCG MERP) index'. ${ }^{6}$ An AE could only be categorised to one grade of severity (table 1).

Two compounded severity categories were constructed in the Norwegian monitoring system in order to make more robust measures; E-I which include all AEs and F-I which include the four most serious categories (table 2). ${ }^{14}$ The category F-I had previously been used in the American study of AEs among Medicare beneficiaries. ${ }^{23}$ Both compounded categories were applied in the comparison of Norwegian and Swedish AE rates.

In Norway, 23 types of AEs were specified (table 3) based on categories adopted from Sweden. AEs were reported in an Excel template, together with supplementary information regarding type, severity and numbering according to admission, so that AEs related to the same admission could be considered and counted 
Table 2 Comparison of adverse event rates according to severity category

\begin{tabular}{|c|c|c|c|}
\hline Severity category & Norway-mean $(95 \% \mathrm{Cl})$ & Sweden-mean $(95 \% \mathrm{Cl})$ & $\begin{array}{l}\text { Sweden minus Norway } \\
\text { difference }(95 \% \mathrm{Cl})\end{array}$ \\
\hline E-I & $12.96 \%(11.68 \%$ to $14.25 \%)$ & $14.41 \%(12.59 \%$ to $16.34 \%)$ & $1.44(-0.82$ to 3.78$)$ \\
\hline F-I & $7.59 \%(6.62 \%$ to $8.68 \%)$ & $7.70 \%(6.71 \%$ to $8.75 \%)$ & $0.11(-1.38$ to 1.56$)$ \\
\hline $\mathrm{E}$ & $6.42 \%(5.67 \%$ to $7.17 \%)$ & $8.10 \%(6.72 \%$ to $9.54 \%)$ & $1.68(0.06$ to 3.34$)$ \\
\hline $\mathrm{F}$ & $6.68 \%(5.70 \%$ to $7.78 \%)$ & $7.01 \%(6.10 \%$ to $7.97 \%)$ & $0.32(-1.09$ to 1.76$)$ \\
\hline G & $0.72 \%(0.53 \%$ to $0.92 \%)$ & $0.42 \%(0.31 \%$ to $0.56 \%)$ & $-0.29(-0.53$ to -0.06$)$ \\
\hline $\mathrm{H}$ & $0.20 \%(0.08 \%$ to $0.33 \%)$ & $0.13 \%(0.08 \%$ to $0.18 \%)$ & $-0.07(-0.22$ to 0.06$)$ \\
\hline I & $0.30 \%(0.21 \%$ to $0.40 \%)$ & $0.28 \%(0.20 \%$ to $0.38 \%)$ & $-0.02(-0.15$ to 0.11$)$ \\
\hline
\end{tabular}

Table 3 Comparison of adverse event (AE) rates in severity category E-I according to type

\begin{tabular}{|c|c|c|c|}
\hline Type of adverse event & Norway (\%) & Sweden (\%) & Sweden minus Norway difference $(95 \% \mathrm{Cl})$ \\
\hline Urinary tract infection & 2.119 & 1.549 & $-0.570(-0.966$ to -0.064$)$ \\
\hline Surgical complication & 1.888 & 0.885 & $-1.003(-1.425$ to -0.4209$)$ \\
\hline Adverse drug event & 1.854 & 1.625 & $-0.229(-0.885$ to 0.473$)$ \\
\hline Postoperative wound infection & 1.624 & 1.588 & $-0.037(-0.385$ to 0.550$)$ \\
\hline Other infection & 1.336 & 1.558 & $0.222(-0.215$ to 0.765$)$ \\
\hline Pneumonia all types & 1.279 & 0.885 & $-0.394(-0.770$ to -0.045$)$ \\
\hline Postoperative haematoma & 0.834 & 0.562 & $-0.272(-0.521$ to 0.026$)$ \\
\hline Reoperation & 0.809 & 0.596 & $-0.213(-0.463$ to 0.071$)$ \\
\hline Pressure ulcer & 0.600 & 1.285 & 0.684 (0.334 to 1.004$)$ \\
\hline Other & 0.435 & 1.036 & 0.601 (0.231 to 1.106$)$ \\
\hline Organ injury & 0.414 & 0.289 & $-0.125(-0.293$ to 0.061$)$ \\
\hline Thrombosis & 0.400 & 0.325 & $-0.075(-0.239$ to 0.089$)$ \\
\hline Falls & 0.372 & 0.756 & $0.384(0.139$ to 0.579$)$ \\
\hline Allergic reaction & 0.306 & 0.259 & $-0.047(-0.162$ to 0.093$)$ \\
\hline Postpartum AE & 0.249 & 0.396 & $0.147(-0.075$ to 0.395$)$ \\
\hline Medical equipment & $0.028^{*}$ & $0.033^{*}$ & $0.005(-0.039$ to 0.045$)$ \\
\hline Ventilator-related pneumonia & 0.084 & 0.137 & $0.053(-0.027$ to 0.178$)$ \\
\hline Central line infection & $0.073^{*}$ & 0.161 & $0.088(0.026$ to 0.189$)$ \\
\hline Wrong-side surgery & $0.000^{*}$ & $0.019^{*}$ & 0.019 (0.004 to 0.042$)$ \\
\hline \multicolumn{4}{|l|}{ Types specified only in Sweden } \\
\hline Distended urinary bladder & & 1.667 & \\
\hline Skin or vessel & & 1.131 & \\
\hline Sepsis & & 0.623 & \\
\hline Vital signs & & 0.438 & \\
\hline Haemorrhage-not surgery & & 0.431 & \\
\hline Anaesthesia-related $\mathrm{AE}$ & & 0.130 & \\
\hline Neurological & & 0.108 & \\
\hline \multicolumn{4}{|l|}{ Types specified only in Norway } \\
\hline Haemorrhage all types & 0.990 & & \\
\hline Postoperative respiratory $A E$ & 0.215 & & \\
\hline Deterioration of chronic condition & 0.183 & & \\
\hline Fractures & 0.069 & & \\
\hline
\end{tabular}

together in the compounded severity categories E-I and F-I. When AEs were reported, the types were not mutually exclusive. If relevant, one $\mathrm{AE}$ could be categorised as more than one type, for example, both a postoperative bleeding and a reoperation. The template was annually sent to the secretariat of the national patient safety campaign. AE rates were also plotted by the
Norwegian hospitals in run charts, which facilitated monitoring.

In Sweden, 26 types of AEs were specified (table 3). Nineteen AE types were specified in both countries giving opportunity for comparisons. The results from each hospital in Sweden were entered into a national database where local results could be compared with 
national average results. The findings of the record review on a national level were presented in a report produced by SALAR. ${ }^{24}$ In addition, the teams in both countries reported the total number of admissions that the investigated records had been randomly selected from. This was used for weighting the team results, when making national estimates.

\section{Ethics}

Approval from research ethics committee was not required or applied for in either country for this data collection and analysis. The Ministry of Health and Care Services in Norway concluded that GTT could be applied as a means of quality assurance at the hospitals within the framework of national regulation, provided that data reported from the hospitals to the national campaign were anonymous. Data reported from hospitals in Norway did not identify individual patients and were evaluated by the Norwegian Data Protection Authority to be anonymous. The study was thus not affected by the Norwegian Health Research Act. In Sweden, the record review was conducted in accordance to national regulation regarding the use of medical records and seen as a part of quality improvement initiatives in the hospitals. Personal identification numbers were not collected or entered into the national database.

\section{Statistical analysis}

Cross-sectional analysis was performed on data from the GTT teams to calculate national AE rates with associated 95\% CIs, divided into types and severities. The national $\mathrm{AE}$ rates were calculated as a weighted average of individual means for the GTT teams. The weight of one GTT team was equal to the number of admissions that investigated records had been randomly selected from for the team, divided by the total number of admissions that investigated records had been randomly selected from for all teams. Non-parametric CIs for the national AE rates were calculated using 10000 bootstrap simulations ${ }^{25}$ since AEs according to type and severity were not symmetrically distributed. The bootstrap was performed by drawing randomly with replacement from the individual means for all 45 GTT teams in Norway and all 63 hospitals in Sweden (each with its own review team). An observation was drawn from the data with probability equal to the weight of the GTT team. For all analyses, the significance level is $5 \%$. The calculations were performed with Microsoft Excel V.2010 and the R statistical software (R: A Language and Environment for Statistical Computing [program]. Vienna, Austria: R Foundation for Statistical Computing, 2015.)

\section{RESULTS}

A total of 10986 records, randomly selected from 569714 admissions in 23 hospitals (with a total of 45 GTT teams) were reviewed in Norway in 2013. This is equivalent to $1.9 \%$ of all eligible admissions. A total of 19141 medical records randomly selected from 1345506 admissions in 63 hospitals (each with its own GTT team) were reviewed in Sweden. This is equivalent to $1.4 \%$ of all eligible admissions. In the samples, a total of 1672 AEs across all severity categories were identified in Norway and 3217 in Sweden. Rates of admissions with one or more AEs in all severity categories are presented in table 2.

There was no significant difference between Norway and Sweden in the rates of hospital admissions with AEs in severity categories E-I, that also include the less severe AEs. Nor was there a significant difference in categories F-I which only include AEs of higher severity, or in category I; AEs that contributed to patient's death. The variation in $\mathrm{AE}$ rates between GTT teams in Norway, and between hospitals in Sweden was large; for E-I: minimum $2.1 \%$ and maximum $21.7 \%$ in Norway and minimum $3.6 \%$ and maximum $31.1 \%$ in Sweden; for F-I: minimum $0 \%$ and maximum $15.0 \%$ in Norway and minimum $0.8 \%$ and maximum $19.3 \%$ in Sweden.

In table 3, rates of different $\mathrm{AE}$ types in severity category E-I are compared. There were significantly higher rates of surgical complications in Norwegian hospitals compared with Swedish hospitals. Swedish hospitals had significantly higher rates of pressure ulcers, falls and 'other' AEs.

In table 4, rates of different types of $\mathrm{AE}$ in severity category F-I are compared. Norwegian hospitals had significantly higher rates of surgical complications than Swedish hospitals. Swedish hospitals had significantly higher rates of postpartum AEs. Eighty-seven per cent of Norwegian AEs of higher severity (F-I) were in the F category, while $90 \%$ of Swedish AEs of higher severity were in the F category.

\section{DISCUSSION}

We have described findings of an explorative study where medical record review with GTT has been used to compare national rates of AEs in acute care hospitals. There are no statistically significant differences between the overall estimates of AEs between the two countries. There are, however, differences in types of AEs between Norway and Sweden. Similar overall results between Norway and Sweden were expected considering the similar conditions and contexts of the two countries' healthcare systems. ${ }^{15}$ The finding that estimated AE rates in Norwegian hospitals correlate with patients' surveyed perceptions of patient safety supports the validity of the results. ${ }^{26}$ Combined, this may indicate that $\mathrm{AE}$ rates produced by GTT may be useful for comparing hospital patient safety between countries. More research on issues like inter-rater reliability and validity across countries is, however, needed before further crosscountry comparison of GTT results is advised. ${ }^{10}$

In Norwegian hospitals, there were significantly higher rates of surgical complications in the combined severity 
Table 4 Comparison of adverse event (AE) rates in severity category F-I according to type

\begin{tabular}{llll}
\hline Type of adverse event & Norway (\%) & Sweden (\%) & Sweden minus Norway difference (95\% Cl) \\
\hline Surgical complication & 1.429 & 0.569 & $-0.860(-1.187$ to -0.420$)$ \\
Postoperative wound infection & 1.204 & 1.403 & $0.199(-0.125$ to 0.737$)$ \\
Adverse drug event & 1.133 & 0.860 & $-0.273(-0.804$ to 0.259$)$ \\
Other infection & 0.893 & 0.814 & $-0.079(-0.334$ to 0.227$)$ \\
Reoperation & 0.762 & 0.550 & $-0.213(-0.460$ to 0.054$)$ \\
Pneumonia all types & 0.728 & 0.605 & $-0.122(-0.424$ to 0.104$)$ \\
Postoperative haematoma & 0.611 & 0.325 & $-0.286(-0.453$ to -0.076$)$ \\
Urinary tract infection & 0.518 & 0.530 & $0.012(-0.211$ to 0.264$)$ \\
Other & 0.343 & 0.597 & $0.254(0.009$ to 0.562$)$ \\
Organ injury & 0.342 & 0.206 & $-0.135(-0.311$ to 0.035$)$ \\
Thrombosis & 0.333 & 0.226 & $-0.107(-0.237$ to 0.046$)$ \\
Falls & 0.148 & 0.182 & $0.034(-0.124$ to 0.132$)$ \\
Allergic reaction & 0.127 & 0.070 & $-0.057(-0.114$ to 0.011$)$ \\
Pressure ulcer & $0.067^{*}$ & 0.243 & $0.176(0.037$ to 0.249$)$ \\
Central line infection & $0.059^{*}$ & 0.078 & $0.019(-0.028$ to 0.084$)$ \\
Ventilator-related pneumonia & $0.024^{*}$ & 0.086 & $0.062(0.016$ to 0.119$)$ \\
Postpartum AE & 0.019 & 0.217 & $0.198(0.101$ to 0.307$)$ \\
Medical equipment & $0.005^{*}$ & $0.018^{*}$ & $0.013(-0.015$ to 0.031$)$ \\
Wrong-side surgery & $0.000^{*}$ & $0.017^{*}$ & $0.017(0.004$ to 0.039$)$
\end{tabular}

Rates (\%) of hospital admissions with at least one AE according to type, severity category F-I combined, in Norway and Sweden in 2013.

*Types with five or less observations greater than zero in Norway or Sweden; the corresponding $\mathrm{Cl}$ must be interpreted with caution.

category E-I, compared with Swedish hospitals. Swedish hospitals had significantly higher rates of pressure ulcers, falls and 'other' AEs. Norwegian hospitals had significantly higher rates of surgical complications also of higher severity (F-I) than Swedish hospitals. Swedish hospitals had significantly higher rates of postpartum AEs of higher severity. It is well known that an increased volume of a specific surgical procedure performed by the same department and team improves medical outcome. In both countries, there are geographical aspects and a question of availability of hospital care that influence the organisation of national health services and the proportion of smaller hospitals as compared with large hospitals and centralised care. Such aspects might contribute to the differences in the amount of AEs related to surgical procedures with higher numbers seen in Norway. The higher rates in Sweden of pressure ulcers, falls and 'other' AEs also indicate that there are differences in the outcome of nursing care between the countries. Differences in the organisation of departments, workload, nursing competence and other factors may have contributed. ${ }^{27}$

Results presented in 'Health at a glance 2013'2 show that obstetric trauma with lacerations of perineum after deliveries with instrument as well as without instrument are more frequent in Sweden than in Norway. This supports the finding of the present study that postpartum AEs (corresponding to lacerations of the perineum) were more common in Swedish hospitals. In Sweden, distended urinary bladder was used as a specific trigger and also did show to be a common type of AE. Late detection of distended urinary bladder has earlier been identified as a frequent $\mathrm{AE}$ in a Swedish orthopaedic clinic and it has also been reported that patients that have suffered distended urinary bladder sometimes have urinary, psychosocial and emotional problems for long afterwards. ${ }^{28}{ }^{29}$ In Norway, distended urinary bladder was not used as a trigger and this type of $\mathrm{AE}$ was classified as 'other' and therefore not possible to identify separately. Prevention of urinary tract infection by restrictive use of urinary bladder catheters were part of both the Norwegian and Swedish patient safety campaigns. The tendency to lower rate of urinary tract infections in Swedish hospitals may reflect a more restrictive use of catheters, which in turn may have entailed an increased rate of distended urinary bladder. We wish to emphasise that the rates of hospitalisations with AEs that contributed to death (severity category I); $0.3 \%$ in both countries, do not reflect deaths that could have been avoided as we did not assess the extent to which the AEs contributed to death nor their preventability.

When interpreting the results in this study, it should be born in mind that the GTT methods used in the two countries were similar but not identical. Both countries adhered to the original definition and method, paying attention to context so that the translated version should be comprehensible. However, in Sweden the initial review in search for triggers in most teams was done by only one instead of two reviewers. This modification could potentially have influenced the results. However, studies report $\kappa$ values from 0.53 to 0.73 on triggers and 0.40 to 0.60 on AEs on agreement between primary nurse reviewers which is fair. ${ }^{12}$ One could also presume that using only one primary reviewer would lead to lower $\mathrm{AE}$ rates of all types. That is not the case in our study; some AE types were more common in Norway and some were more common 
in Sweden. We therefore consider that modifying the reviewing process in Sweden probably has had minor influence on the results. Still we recommend avoiding any deviation from the original reviewing method in future cross-country comparisons using the GTT.

Cross-country comparisons based on medical record review require that the samples reviewed are representative for the type of hospital care in each country. In this study, the numbers of records reviewed are probably the largest random samples of admissions drawn from all hospitals in two countries that have been reviewed with GTT. That should with reason be sufficient for comparing overall results as well as specific AE type rates. Unfortunately, data protection regulations did not allow us to collect individual demographic or other patient data, which would have allowed us to identify and correct for possible differences in characteristics between the populations of the two countries which could have influenced the results. However, the risk for such differences is probably minor as the general demographics and other population characteristics of the two countries are very similar. The variation in $\mathrm{AE}$ rates was large between GTT teams in Norway, and between hospitals in Sweden. That is expected since rates are based on cross-sectional analyses of small samples allowing large random variation. In addition, there are differences between characteristics of hospitals' patient populations and activities. For this reason, we do not use GTT results for comparison between hospitals.

No previous studies have to the best of our knowledge compared national rates of hospital AEs between countries based on the GTT. Other studies have presented results for regions and healthcare systems, ${ }^{14} 30$ as well as subgroups at national level. ${ }^{23}$ International comparisons of levels of patient safety are of great interest as a means to study organisational development in healthcare. ${ }^{2} 32$ Comparison based on nationwide data should, however, be interpreted with caution and in order to improve measures and data quality for international comparisons, several methods have been developed. ${ }^{1} 4$ Presently, there is no generally accepted gold standard for such comparisons. Agency for Healthcare Research and Quality (AHRQ) and OECD indicators based on hospital discharge data are used but some indicators remain unreliable for international comparisons due to varying data quality, undercoding and lack of precision in documentation. Furthermore, there are differences in International Classification of Diseases (ICD) coding guidelines and practices, and in many countries coding is influenced by financial incentives. ${ }^{3}$ In a recent publication, it is argued that the current version of the ICD, the 10th revision, is not optimal for the capture of healthcare-related harm and injury events which has resulted in the development of a new framework for the coding and capture of healthcare-related harm. ${ }^{33}$ ICD codes that better identify harm could be a good supplement to medical record review with GTT, since ICD codes also can be used as triggers.

\section{CONCLUSIONS}

AE rates as assessed by GTT may be useful for comparing hospital patient safety between countries. In our explorative study, the overall level of patient safety in acute care hospitals was essentially the same in both countries. This is expected considering similarities between the two countries' healthcare systems. The difference between the countries in the rates of several types of AEs merit further investigation and provide new incentives for Norwegian and Swedish governing bodies to address patient safety issues.

\section{Author affiliations}

${ }^{1}$ National Patient Safety Program, Norwegian Directorate of Health, Oslo, Norway

${ }^{2}$ Health Services Research Center, Akershus University Hospital, Lørenskog, Norway

${ }^{3}$ Center for Healthcare Development, County Council of Östergötland, Sweden ${ }^{4}$ Norwegian Computing Center, Oslo, Norway

${ }^{5}$ National Board of Health and Welfare, Stockholm, Sweden

${ }^{6}$ Division of Health Care Analysis, Department of Medical and Health Sciences, Linköping University, Linköping, Sweden

${ }^{7}$ Swedish Association of Local Authorities and Regions, Stockholm, Sweden

Acknowledgements We thank GTT teams in all the hospitals for their contributions to the study. We thank the referees for valuable comments, which have led to great improvements to the quality of the paper.

Contributors ETD was responsible for designing the study in Norway, training of reviewer teams, data collection, analysis and interpretation of data and writing the manuscript. MBR contributed with Swedish data collection and in their interpretation. MH participated in data collection in Norway, in the statistical analysis and interpretation, in addition to critically revising the manuscript. JCL participated in the statistical analysis and interpretation, in addition to revising the manuscript. UN participated in designing the study in Sweden, training of reviewer teams, data collection, analysis and interpretation of data, and revising the manuscript critically. HR participated in designing the study in Sweden, training of reviewer teams, data collection, analysis and interpretation of data and revising the manuscript critically. MS participated in designing the study in Sweden, analysis and interpretation of data and revising the manuscript critically. All authors read and approved the final manuscript.

Funding No grants were specifically awarded for this research. Reviewer teams were funded by the hospitals. The research was funded by Akershus University Hospital's Health Services Research Unit, the Norwegian Computing Center and by the Swedish Association of Local Authorities and Regions (SALAR).

Competing interests None declared.

Provenance and peer review Not commissioned; externally peer reviewed.

Data sharing statement The data are administered by the Norwegian Patient Safety Program, at the Norwegian Directorate of Health and by the Swedish Association of Local Authorities and Regions (SALAR). No additional data are available.

Open Access This is an Open Access article distributed in accordance with the Creative Commons Attribution Non Commercial (CC BY-NC 4.0) license, which permits others to distribute, remix, adapt, build upon this work noncommercially, and license their derivative works on different terms, provided the original work is properly cited and the use is non-commercial. See: http:// creativecommons.org/licenses/by-nc/4.0/

\section{REFERENCES}

1. Mainz J, Bartels $P$, Rutberg $H$, et al. International benchmarking. Option or illusion? Int J Qual Health Care 2009;21:151-2. 
2. OECD. Health at a Glance 2013: OECD Indicators. Health at a glance, 2013.

3. Drösler SE, Klazinga NS, Romano PS, et al. Application of patient safety indicators internationally: a pilot study among seven countries. Int J Qual Health Care 2009;21:272-8.

4. Nordisk Ministerråd. Nordisk kvalitetsmåling i sundhedsvæsenet. København, Denmark, 2010:119-23.

5. Working group on Patient Safety under Nordic Ministry Council. $A$ report on patient safety. Copenhagen: Styrelsen for Patientsikkerhed, 2016.

6. Griffin F, Resar R. IHI global trigger tool for measuring adverse events IHI innovation series. 2nd edn. Cambridge, MA: Institute for Healthcare Improvement; 2009.

7. Doupi P, Svaar H, Bjørn B, et al. Use of the Global Trigger Tool in patient safety improvement efforts: Nordic experiences. Cogn Tech Work 2015:17-45.

8. Health Foundation. Evidence scan: global trigger tools. London, UK The Health Foundation, 2010.

9. Sharek PJ, Parry G, Goldmann D, et al. Performance characteristics of a methodology to quantify adverse events over time in hospitalized patients. Health Serv Res 2010;46:654-78.

10. Hanskamp-Sebregts $M$, Zegers $M$, Vincent C, et al. Measurement of patient safety: a systematic review of the reliability and validity of adverse event detection with record review. BMJ Open 2016;6: e011078.

11. Health Quality \& Safety Commission. The global trigger tool: a review of the evidence-report for the health quality \& safety commission New Zealand. Wellington: Health Quality \& Safety Commission, 2013.

12. Good VS, Saldaña M, Gilder R, et al. Large-scale deployment of the Global Trigger Tool across a large hospital system: refinements for the characterisation of adverse events to support patient safety learning opportunities. BMJ Qual Saf 2011;20:25-30.

13. Rutberg $\mathrm{H}$, Borgstedt Risberg M, Sjødahl R, et al. Characterisations of adverse events detected in a university hospital: a 4-year study using the Global Trigger Tool method. BMJ Open 2014;4: e004879.

14. Deilkås E, Bukholm G, Lindstrøm JC, et al. Monitoring adverse events in Norwegian hospitals from 2010 to 2013. BMJ Open 2015;5:e008576.

15. Veggeland Noralv. The current Nordic welfare state model. New York: Nova Science Pub Inc, 2016.

16. Magnussen J, Vrangbaek, K, Saltman RB. Nordic health care systems-recent reforms and current policy challenges. Berkshire, UK: Mc Graw Hill Open University Press, 2009.

17. Soop M, Fryksmark U, Köster M, et al. The incidence of adverse events in Swedish hospitals: a retrospective medical record review study. Int J Qual Health Care 2009;21:285-91.

18. The Norwegian patient safety campaign secretariat. Final report. Secondary Final report 2014. http://www.
pasientsikkerhetsprogrammet.no/no/l+trygge+hender/L\%C3\%A6r +om+programmet/ attachment/2925? ts=146d6eb36d2

19. Patientsäkerhetssatsning 2011 överenskommelse mellan staten och Sveriges Kommuner och Landsting om förbättrad patientsäkerhet. In: Government TS, ed. Stockholm, 2011.

20. Den nasjonale pasientsikkerhetskampanjen. Strukturert journalundersøkelse, ved bruk av Global Trigger Tool for å identifisere og måle forekomst av skader i helsetjenesten, 2010.

21. Sveriges Kommuner och Landsting. Markörbaserad journalgranskning - för att identifiera och mäta skador i vården. LTAB: Sveriges Kommuner och Landsting, 2012.

22. Sveriges Kommuner och Landsting. Markörer med definitioner Stockholm: Sveriges Kommune og Landsting, 2012

23. Levinson DR. Adverse events in hospitals. National incidence among Medicare beneficiaries. Department of Health and Human Services, Office of Inspector General, 2010.

24. Sveriges Kommuner och Landsting. Vårdskador VAD TRODDE VI $D A ̊-V A D$ VET VI NU? Stockholm: Sveriges Kommuner och Landsting, 2016.

25. Efron B, Tibshirani RJ. An introduction to the bootstrap. London: Chapman \& Hall, 1993

26. Bjertnæs $\varnothing$, Skudal KE; Iversen $\mathrm{HH}$, et al. The Patient-Reported Incident in Hospital Instrument (PRIH-I): assessments of data quality, test-retest reliability and hospital level reliability. BMJ Qual Saf 2013;22, 743-51.

27. Aiken LH, Sermeus W, Van den Heede K, et al. Patient safety, satisfaction, and quality of hospital care: cross sectional surveys of nurses and patients in 12 countries in Europe and the United States. BMJ 2012;344;e1717.

28. Unbeck M, Dalen N, Muren O, et al. Healthcare processes must be improved to reduce the occurrence of orthopaedic adverse events. Scand J Caring Sci 2010;24:671-7.

29. Joelsson-Alm E, Nyman CR, Svensé et al. Micturition problems after bladder distension during hospitalization in Sweden: "I'm not ill, just damaged for the rest of my life". Nurs Res 2014;63:418-25.

30. Landrigan CP, Parry GJ, Bones CB, et al. Temporal trends in rates of patient harm resulting from medical care. $N$ Engl J Med 2010;363:2124-34.

31. Garrett PR Jr., Sammer C, Nelson A, et al. Developing and implementing a standardized process for global trigger tool application across a large health system. J Comm J Qual Patient Saf 2013;39:292-7.

32. Braithwaite J, Matsuyama Y, Mannion R, et al. Healthcare Reform, Quality and Safety: perspectives, participants, partnerships, and prospects in 30 countries. Dorchester: Ashgate, 2015.

33. Southern DA, Pincus HA, Romano PS, et al. Enhanced capture of healthcare-related harms and injuries in the 11th revision of the International Classification of Diseases (ICD-11). Int J Qual Health Care 2015. 